\title{
Resistance of Alcat1 Null Mice to High Fat Diet Induced Obesity and Impaired Glucose Tolerance
}

\author{
Yan Yan (Corresponding author) \\ Intercollege Program in Genetics, College of Medicine \\ The Pennsylvania State University, Hershey, Pennsylvania 17033 \\ $\&$ \\ Cell and Developmental Biology Program \\ The Pennsylvania State University, University Park, Pennsylvania 16801 \\ Tel: 717-343-2307 E-mail: yanyanms@gmail.com \\ Bin Kang \\ Department of Biology, University of Pennsylvania, Pennsylvania 19104
}

Received: October 12, 2010 Accepted: October 29, 2010 doi:10.5539/ijb.v3n2p11

The research is financed by Penn State University

\begin{abstract}
The mouse acyl CoA: lysocardiolipin acyltransferase (ALCAT1) exhibited lysocardiolipin acyltransferase activity and polyglycerophospholipid acyltransferase activity in vitro and therefore was predicted to play a role in polyglycerophospholipid remodeling pathway. To investigate the physiological function of ALCAT1 in vivo, alcat 1 null mice were generated. The alcat 1 null mice were challenged with $60 \mathrm{kcal} \%$ high fat diet (HFD) to examine ALCAT1's effects on the whole body energy metabolism such as the body weight, tissue content and blood glucose level. Interestingly, the null mice gained less body weight than the controls, and exhibited less fat tissue content while the food consumptions were similar in both mice groups. Lower fasting blood glucose level and less impaired glucose tolerance test in null mice were observed after HFD challenging. Besides, the yeast complementation experiment also suggested that ALCAT1 might function through its acyltransferase activities in vivo. In summary, this study suggests that ALCAT1 plays a critical role in regulating energy balance in vivo.
\end{abstract}

Keywords: Acyl CoA, Lysocardiolipin acyltransferase (ALCAT1), Knock out; high fat diet (HFD), Obesity, Impaired glucose tolerance

\section{Introduction}

Cardiolipin belongs to the family of polyglycerophospholipids and constitutes $20 \%$ of total lipid of mitochondria inner membrane (Schlame et al. 2000; Hatch, 1998). Polyglycerophospholipids remodeling is a key step for cardiolipin and other polyglycerophospholipids to obtain proper acyl chains in vivo. The acyl chain content is very important for the physiological function of cardiolipin and other phospholipids, such as maintaining of the membrane potential and resistance to oxidative stress (Schlame et al. 2000; Hatch, 1998; Bogdanov et al. 2008; Sen et al. 2007; Schlame et al. 2009). The mechanism of cardiolipin remodeling is still elusive. The Land's cycle occurring in microsomes is the best-known process of phospholipids remodeling via deacylation and reacylation so far (Eichberg et al. 1974). A mouse microsomal enzyme acyl CoA: lysocardiolipin acyltransferase (ALCAT1) was found to possess the acyltransferase activity in the polyglycerophospholipids remodeling (Cao et al. 2004; Cao et al. 2009). Multiple ALCAT1 orthologs were identified from human to fish, except Caenorhabditis elegans and Saccharomyces cerevisiae. Northern Blotting analysis showed ALCAT1 highly expressed in mouse muscles, heart and liver. ALCAT1 contains an endoplasmic reticulum (ER) localization motif (KKXX) at its C-terminus and the recombinant ALCAT1 was found to localize in ER of mammalian cells (Cao et al. 2004).

ALCAT1 contains a highly conserved phosphate acyltransferase domain (Plsc), which is also found in the TAZ protein that shows lysocardiolipin acyltransferase activity involved in cardiolipin remodeling (Cao et al. 2004; 
Neuwald, 1997). Wide ranges of phospholipids substrates of ALCAT1 were identified in vitro so far. ALCAT1 exhibited substrate specificity for monolysocardiolipin (MLCL), dilysocardiolipin(DLCL), and other members of polyglycerophospholipid including phosphatidylglycerol (precursor of cardiolipin synthesis), and bis (monoacylglycerol) phosphate (an isomer of lysophosphatidylglycerol and a intermediate of cardiolipin) in vitro. ALCAT1 is a CoA dependent enzyme and can incorporate 18:2 acyl chain and other polyunsaturated moieties into polyphospolipid (Cao et al. 2004; Cao et al. 2009). The study of ALCAT1 protein structure indicated that D168 and L169 are critical amino acids potentially involved in its lysophospholipid substrate binding. Damage of cardiolipin content and species caused different type of metabolism situations, such as Barth Syndrome, heart failure, and aging (Zhao et al. 2009). The cardiolipin content is linked to the mitochondrial functions of oxidative phosphorylation (Schlame et al. 2000; Hatch, 1998; Bogdanov et al. 2008; Sen et al. 2007; Schlame et al. 2009).

It has been reported that high fat diet caused serious diseases including obesity, hypertension and early diabetes (Holl et al. 2010; Waqar et al. 2010; Hur et al. 2010). The early stages of diabetes, such as impaired glucose tolerance (IGT) and impaired fasting glucose (IFG) are common in USA population. About 10 to 15 percent of adults in the United States show one of above conditions. The impaired glucose tolerance is also a risk factor of mortality. Therefore people with impaired glucose tolerance and impaired fasting glucose become an important target group for primary prevention of diabetes (Rao et al. 2002; Barr et al. 2007). The studies of ALCAT1 in vitro suggested that ALCAT1 might play a role in cardiolipin and polyglycerophospholipids remodeling. ALCAT1 exhibits an enzyme activity effectively on a wide range of substrates (polyunsaturated acyl chains and phospholipids) including lysocardiolipin. However, the physiological function of ALCAT1 in vivo is largely unknown. To explore how ALCAT1 functions in mouse energy metabolism, a new genetics model of alcat 1 null mouse was generated and the null mice showed stronger catabolic metabolism and resistance to high fat diet induced obesity. Therefore these results suggested ALCAT1 possible positively regulated the anabolic metabolism in vivo.

\section{Material and Experiment Methods}

\subsection{Animals}

The animals were maintained and handled according to standard animal experimental protocol 2006-053 approved by PSU-COM Institutional Animal Care and Use Committee. Two breeding pairs of alcat 1 knock out mouse (KO) (C57/BL6) (generated by Lilly Research Laboratories, Eli Lilly and Company, Indianapolis, IN) were transferred from Taconic (Taconic Farms, Inc.) and were housed in standard barrier facilities free of pathogens (Department of Comparative Medicine, PSU-COM). Null mouse founder was backcrossed with wild type (C57/BL6) prior to breeding of the study groups. All animals were maintained on a 12/12 hour light/dark cycle. Every cage housed no more than five mice. Food and water were given ad libitum. Control mice $(-/+)$ were littermates of knockout mice $(-/-)$.

\subsection{Identification of Knockout alcat1 Mice with PCR genotyping}

The alcatl knockout mice were generated following the standard protocols (Hogan, et al., 1994) (Lilly Research Laboratories). The experimental mice were generated from breeding of heterozygous mice and the genotype of progenies was identified by PCR genotyping. PCR genotyping was performed according to the protocol provided by Taconic. Briefly, PCR template, the mice genomic DNA, was isolated from mice tail using a genomic DNA purification System (Promega). Taconic provided the primers' sequence for alcat1 null mice PCR genotyping and the primers were synthesized in Integrated DNA Technologies (IDT). The primers YS104W1 and YS104S1 are for detecting the wild type allele; the primers N752S and N1131AS are for detecting the knock out allele. Wild type allele generates a 558bp fragment; while the knock out allele generates a $375 \mathrm{bp}$ fragment. PCR was performed using Taq polymerase (Promega).

\subsection{RNA Isolation and Northern Analysis}

Total RNA was isolated from mouse liver using the Trizole protocol (Invitrogen). 10-30ug total RNA per sample was subjected to electrophoresis, and RNA samples were blotted overnight to a Nylon membrane (Roche) (Sambrook and Russell, 2001). ALCAT1 sequence was used as the probe; GAPDH was used as an internal control (a gift from Dr Ji-Yue Zhu's lab, PSU-COM). Probes were radio labeled with $\left[\alpha^{32} \mathrm{P}\right] \mathrm{dCTP}$ (Perkin Elmer) by random primer labeling kit (Invitrogen). The autoradiography was performed by the Typhoon Imager (GE Healthcare Bio-Sciences) and was quantified by ImageQuant software program.

\subsection{High Fat Diet Challenging and food consumption}

$60 \%$ fat (kcal \%) Rodent Diet (Research Diets, catalogue number: D12492) was used. Knockout mice and control mice, 4-6 weeks old, were provided with high fat diet food ad libitum for 8 weeks. Food weights of each cage were 
measured every week before mice feeding and after feeding. The average food consumption per mouse in a week was calculated as:

Average Food consumption $(\mathrm{g} /$ week / mouse $)=($ food weight before a week of feeding - leftover food weight after a week of feeding)/ number of mouse per cage

\subsection{Body Weight and Tissue Content Measurements}

Body weight measurements were performed respectively before high fat diet challenging and at 2, 4, 5, 6, 7 or 8 weeks with high fat diet challenging. Every mouse was measured individually on an electronic scale (Denville Scientific). After mice were fed with high fat diet for 8 weeks, tissue contents were measured at Mouse Metabolic Phenotyping Center (PSU-COM). Fat tissue/lean tissue/body fluids were measured by EchoMRI 3-in-1 (Echo Medical System) for the un-anesthetized mice.

\subsection{Oral Glucose Tolerance Test}

Oral Glucose Tolerance Test (GTT) was performed before $60 \%$ high fat diet feeding and 8 weeks later after high fat diet feeding, respectively. Mice were fasted for 16-18 hours before the day of the experiment. Unanesthetized mice were orally fed $50 \%$ D-glucose with the amount of $1 \mathrm{~g} / \mathrm{kg}$ body weight with 10 "gauvage. Blood glucose were measured from tail vein by One-touch glucose meter and One-touch test strips (LifeScan) at time 0,30, 60, 90, and 120 minutes after glucose administration.

\subsection{Yeast complementation}

Complementation experiment was performed as previously described with a little revision (Schmidt et al. 1997). In brief, the $\Delta t a z$ and wild type strains (w303) provided by Euroscarf (Euroscarf, German) were transformed with vector pEMBLyes4 with/without ALCAT1 gene. Positive clones were cultivated in selective liquid SD-URA medium for overnight at $30^{\circ} \mathrm{C} .1 \mathrm{OD}_{600}$ cells were washed with $1 \mathrm{x}$ phosphate buffer saline (PBS) and suspended into 100ul 1x PBS. 1-5ul sequential 10x diluted cell was doted on the agar plate of URA selective SD medium with $2 \%$ galactose. Plates were incubated in $37^{\circ} \mathrm{C}$ incubator for 2 days before observing results and taking picture.

\subsection{Statistical Analysis}

Results were presented as mean \pm standard error of the mean (SEM). Results were compared using Student's t-test to assess differences between two genotype groups or two treatment groups. Differences were considered significant when $\mathrm{P}<0.05$.

\section{Results}

\subsection{Identification of alcat1 knockout mouse}

According to PCR genotyping (Fig 1A), ALCAT1 expression was examined in liver tissues of mice with different genotypes. Consistent with PCR genotyping, Northern-blotting showed different ALCAT1 mRNA levels in liver tissues of mice with different genotypes (Fig 1B). Clearly, there was no ALCAT1 mRNA detected in null mice (-/-); less ALCAT1 mRNA was present in heterozygous mouse $(-/+)$ than in wild type $(+/+)$; while the internal control of Glyceraldehyde3-Phosphate Dehydrogenase (GAPDH) showed same sample loading in all groups.

\subsection{The alcat1 null mouse showed a smaller body weight than wild type challenged by high fat diet (HFD)}

The high fat diet D12492 (Research diets) provides 60\% of total energy through diet fat (supplementary data 2). It has been reported that high fat diet D12492 effectively induced phenotypes of obesity and diabetes in C57BL/6 wild type mice (Bush et al., 2001; Shapiro, 2001; Parekh et al., 1998). Therefore, we chose this diet to perform the diet challenging on the alcat 1 null mice, which is in the C57BL/6 background as well. Since there were no different responses identified between heterozygous and wild type littermates to the high fat diet challenging (supplementary data 1), alcat 1 knockout mutation is recessive, thus the heterozygous littermates (+/-) were used as negative controls of alcatl null (-/-) mice in all the subsequent experiments.

In male mice, the body weight of alcat 1 null mice $(29.6 \mathrm{~g} \pm 0.4)$ had been significant less than control mice (31.1 $\mathrm{g} \pm 0.4$ ) since the $2^{\text {nd }}$ weeks' HFD feeding (Student's t-test, $* * \mathrm{P}<0.01$ ) (Fig. $2 \mathrm{~A}$ ). Moreover, the difference of body weight between null mice and control mice increased dramatically; the body weight of control mice reached $48.3 \mathrm{~g} \pm 0.4$, while null mice was only $41.4 \mathrm{~g} \pm 1.5$ in the $8^{\text {th }}$ week of HFD feeding (Student's t-test, $* * \mathrm{P}<0.01)$ (Fig2A). However, the negative control $(19.4 \mathrm{~g} \pm 0.2)$ showed similar body weight to that of null males (19.8g \pm 0.2$)$ before HFD (Fig. 2A).

The effect of HFD was not so quickly observed in female mice (Fig. 2B). The two groups of female mice showed slight difference in body weight during seven weeks of high fat diet feeding. However, the null female 
mice exhibited much smaller body weight than controls in the $8^{\text {th }}$ week of HFD feeding.

\subsection{The alcat 1 null mice showed lower fat tissue content after challenged by high fat diet}

Within eight weeks of HFD, the male null mice showed $21.9 \%$ less fat tissue $(17.8 \mathrm{~g} \pm 0.48)$ than the control mice $(21.7 \mathrm{~g} \pm 0.53)$ (Student's t-test, $* * \mathrm{P}<0.01)$, while there was small difference of the lean tissue between null mice $(22.1 \mathrm{~g} \pm 1.2)$ and control mice $(25.0 \mathrm{~g} \pm 0.2)$ (Fig. $3 \mathrm{~A})$. Also the male null mice $(0.07 \%)$ contained much less water than control mice $(0.2 \%)$ (Student's t-test, $* * \mathrm{P}<0.01)$ (Fig. 3B). Besides, the percentage of lean tissue $\left(\mathrm{W}_{\text {lean }} / \mathrm{W}_{\text {body }}\right.$ in male null mice $(55 \%)$ was a slightly more than that of male control $(53 \%)$. The female null $(12.5 \mathrm{~g} \pm 2.2)$ showed lower fat weight than the female controls $(17.5 \mathrm{~g} \pm 2.1)$, yet the difference was not statistically significant (Fig. 3C, 3D). And lower percentage of fat tissue $\left(\mathrm{W}_{\mathrm{fat}} / \mathrm{W}_{\mathrm{body}}\right.$ in null female $(40 \%)$ than the controls $(47 \%)$ was observed.

\subsection{The high fat diet food consumption of null mice was similar to the control mice}

Body weight differences between alcat 1 null mice and control mice could be attributed to the food consumption difference between mice groups. To test the hypothesis, food consumptions were measured in different genotypes and different genders. However, the food consumptions varied slightly in each group and each week (Table1). Though the null mice consumed a little more than the controls, there were no significant differences in food consumption between two groups.

3.5 The alcat1 null mice showed better fasting blood glucose level and oral blood glucose tolerance test (GTT) than controls after HFD treatment

It was found that the fasting blood glucose level of the male control mice $(240 \mathrm{mg} / \mathrm{dL} \pm 14.5)$ elevated much higher than that of male null mice $(158 \mathrm{mg} / \mathrm{dL} \pm 7.1)$ (Student's t-test, $* * \mathrm{P}<0.01)$ after HFD feeding, although the two groups showed similar low fasting blood glucose before HFD $(88 \mathrm{mg} / \mathrm{dL} \pm 3.9$ and $86 \mathrm{mg} / \mathrm{dL} \pm 2.5$, respectively) (Fig 4A, 4B). Likewise, the elevation of fasting blood glucose level of female control $(245 \mathrm{mg} / \mathrm{dL} \pm$ 30.1) was two folds higher than that of female null mice $(96 \mathrm{mg} / \mathrm{dL} \pm 5.9)$ with HFD feeding (Student's t-test, $* * \mathrm{P}<0.01$ ) (Fig. 4D), the fast blood glucose before HFD was still alike between control ( $74 \mathrm{mg} / \mathrm{dL} \pm 3$ ) and null mice $(78 \mathrm{mg} / \mathrm{dL} \pm 6)($ Fig. $4 \mathrm{C})$. It also showed that the level of fasting blood glucose of female mice was lower than that of male mice during the process.

Consequently, null mice exhibited better performance in blood glucose tolerant test than control mice of both male and female after HFD feeding. A much lower blood glucose record $\left(340 \mathrm{mg} / \mathrm{dL} \pm 34,30^{\prime}\right)$ was found in male null mice, though the male control had reached $553 \mathrm{mg} / \mathrm{dL} \pm 13\left(30^{\prime}\right)$ (Student's t-test, ${ }^{*} \mathrm{P}<0.05,{ }^{*} * \mathrm{P}<0.01$, $30^{\prime}$ ) (Fig 4B). There was no much difference of GTT between the male control mice ( $\left.269 \mathrm{mg} / \mathrm{dL} \pm 12,30^{\prime}\right)$ and the null male mice $(260 \mathrm{mg} / \mathrm{dL} \pm 20,30$ ') before HFD feeding (Fig 4A). Likely, in GTT test after HFD, the female control demonstrated much higher blood glucose $\left(482 \mathrm{mg} / \mathrm{dL} \pm 30,30^{\prime}\right)$ than that of female null (239 $\mathrm{mg} / \mathrm{dL} \pm 22,30$ ') (Student's t-test, $\left.{ }^{*} * \mathrm{P}<0.01\right)$ (Fig $4 \mathrm{D}$ ), while the female control (282 $\mathrm{mg} / \mathrm{dL} \pm 30,30^{\prime}$ ) was only little higher than that of female null (204mg/dL $\pm 22,30$ ') before HFD feeding (Fig 4C).

3.6 Mouse ALCAT1 protein complemented the growth retardation of $\triangle$ taz1 temperature mutant in non-permissive temperature

Mouse ALCAT1 shares a conserved Phosphate acyltransferases (Plsc) domain with yeast Tazlp and both of them showed acyltransferases activity. There is no ALCAT1 ortholog in yeast (Cao et al. 2004; Gu et al. 2004; Testet et al. 2005). Protein BLAST was performed between mouse ALCAT1 protein and yeast Taz1p protein in amino acids sequences and found four alignments of the two proteins with low but significant similarity scores. The Plsc domain got the highest alignment score among the four alignments in the blast, suggesting the highly conservation of amino acid sequence between two proteins. Loss function of tazl in yeast showed a temperature sensitive phenotype that the cell growth was slowed at $37^{\circ} \mathrm{C}$ due to the compromised membrane stability of mitochondria (Testet et al. 2005; Ma et al. 2004). Expression of mouse ALCAT1 in Atazl yeast mutant partially rescued the growth retardation at non-permissive temperature, $\Delta$ tazl yeast mutant clearly showed growth defect at $37^{\circ} \mathrm{C}$ (Fig 5). Six independent complementation experiments were performed and consistent results were observed.

\section{Discussion}

Mouse ALCAT1, an acyl-CoA acyltransferase, functions in the cardiolipin and other polyglycerophospholipids remodeling pathway in vitro (Cao et al. 2004; Cao et al. 2009). In order to know the role of ALCAT1 in energy balance of the mouse whole body, alcat 1 knockout mice were generated. In consistent with PCR genotyping (Fig. 1A), Northern blotting showed that deletion of $1^{\text {st }}$ exon of ALCAT1 gene completely abolished the ALCAT1 mRNA expression in homozygous mice in vivo (Fig. 1B). 
It has been reported that the $60 \mathrm{kcal} \%$ HFD applied in this study can effectively induce obesity in wild type male mice (C57/B16) (Bush et al. 2001; Shapiro, 2001; Parekh et al. 1998). The control male mice showed body weight gaining as wild type littermates when fed with this HFD (supplementary data 1) suggesting that alcat1 knockout mutation is recessive. Surprisingly, challenged by the same HFD, alcat1 null male mice exhibited less body weight gaining, less fat tissue content than control males (Fig.2A, Fig.3A, 3B), although their food consumption was found to be close to control mice (Table 1). Similarly, null male mice showed significant less fat tissue and water weight than control mice, while null mice and control mice were similar in their lean tissue contents (Fig.3A, 3B). Clearly, the difference of body weight between null and control mice were mainly attributed to the difference of their fat tissue contents. These results suggested that loss of the function of ALCAT1 caused less energy deposit such as fat store in vivo, which may due to high level of thermogenesis, physical activities, and obligatory energy expenditure in vivo (Bradford et al. 2000).

The fact that male null mice contained more percentage of the lean tissue than the controls indicates more energy expenditure in null mice (Fig2B, 2D). The mitochondrion enriched skeleton muscle in the lean tissue is one of the key factors affecting the energy expenditure: the skeleton muscle is positive associated with the whole body energy expenditure even in resting status (Zurlo et al. 1990). Mitochondria in muscle could increase oxidative phosphorylation (OXPHOS) to adapt to the transient high fat diet feeding (within 14 days) and thus limit fat deposition in soleus. However, long-term high fat diet can cause significant decrease in oxidative phosphorylation (OXPHOS) activity in rat muscle (Chanseaume et al. 2007). The mechanism of ALCAT1 affects the function of mitochondria in vivo is not clear yet, it is clear that cardiolipin plays key roles in oxidative phosphorylation, apoptosis and other function of mitochondrial energetic generation (Schlame et al. 2000; Bogdanov et al. 2008; Schlame and Ren, 2009). Depletion of cardiolipin content and species have been known to be linked to mitochondria oxidative stress and aging response in vitro, which indicated the potential of mitochondrial inner membrane was lost and may lead to oxidative phosphorylation uncoupling, depletion of ATP and activation of apoptotic cascade in the tissue (Sen et al. 2007; Schlame and Ren, 2009). Moreover, loss function of mouse phospholipase PLA2 gama which was involved in the cardiolipin metabolism was reported to be resistant to high fat diet induced obesity and insulin resistance in vivo. The iPLA2gama (-/-) knockout mice showed significantly decrease in State 3 respiration of skeleton muscle due to the uncoupling of phosphorylation and oxidative. Moreover, the iPLA2gama (-/-) knockout mice kept an increased fatty acid oxidation in adipose tissue under long term high fat diet treatment (Mancuso et al. 2010; Malhotra et al. 2009; Zachman et al. 2010). Therefore, it is quite possible that ALCAT1 negatively affects mouse whole body energy expenditure through its indirect disturbance of the process of oxidative phosphorylation of mitochondria.

The alcat1 null mice exhibited a better performance in GTT and fasting blood glucose level when challenged with high fat diet indicating its resistance to HFD induced hyperglycemia (Fig.4). The elevation of fast blood glucose and impaired glucose tolerance are the signs of pre-diabetes or hyperglycemia (Rao et al. 2002). It was reported that this kind of high fat diet could induce obesity accompanied with insulin resistance and elevated blood glucose level in wild type male mice. Due to high fat diet, the mitochondria dysfunction leads to hyperglycemia, insulin resistance and other disorder of metabolism such as diabetes, heart failure and oxidative stress, which was reported in many researches (Holl et al. 2010; Waqar et al. 2010; Jornayvaz et al. 2010; Hur et al. 2010; Bush et al. 2001; Shapiro, 2001; Parekh et al. 1998). However, loss function of iPLA2 gama in mouse causing cardiolipin content decrease in muscle mitochondria exhibited insulin sensitive and normal glucose tolerance phenotype in HFD feeding (Mancuso et al. 2010; Malhotra et al. 2009; Zachman et al. 2010). The alcat Inull mice (both male and female) showed close to normal glucose tolerance test (GTT) and fasting blood glucose level (both male and female) after HFD feeding (Fig 4), suggesting alcat1 null mice were insulin sensitive and may not impair the fatty acid oxidization and function of insulin receptors (Bush et al. 2001; Shapiro, 2001; Parekh et al. 1998).

This report also showed gender difference of mice in their response to HFD feeding. Generally speaking, regarding body weight and fat tissue content, male mice were more sensitive to HFD while female mice were not (Fig. 2, Fig. 3); and the male fasting blood glucose level and GTT test were more sensitive to HFD than those of female (Fig. 4). These results are consistent with the previous finding that the higher efficiency of energy expenditure in female than male in mice was due to the effect of sex hormone (Dubuc et al. 1993; Wiedmer et al. 2004).

As to the biochemical function of ALCAT1 in vivo, the complementation experiment showed ALCAT1 partially complemented the function of yeast Taz1p in vivo (Fig.5). The yeast tazl gene is well known homologue of human taffazzin whose mutation is tightly linked to the Barth Syndrome; loss function of tazlp in yeast caused mitochondria membrane instability (Testet et al. 2005; Ma et al. 2004; Ma et al. 2004). So our complementation 
result suggested that it was very likely that ALCAT1 functioned as an Acyl-CoA acyltransferase in vivo. The low but significant alignment score in the Plsc domain between ALCAT1 and yeast Tazlp suggested function conservation of the Plsc domain of the two genes. ALCAT1 partially complemented the stability defects of mitochondria membrane in $\triangle t a z$ mutant indicating that ALCAT1 could be involved in phospholipids content of mitochondria membrane such as polyglycerophospholipid remodeling. Since loss function of ALCAT1 could change the profile of phospholipids in vivo, including cardiolipin and other lipid $2^{\text {nd }}$ messengers, which would therefore affect the potential gradient across the mitochondrial inner membrane, destabilize the electronic transport chain complexes, uncouple oxidative phosphorylation and reduce ATP synthesis, thus whole body energy generation would be potentially decreased (Lamson et al. 2002; Ritz and Berrut, 2005; Schlame and Ren, 2009). Due to the possibility of less usable energy generated in mitochondria, alcat1 null mice gained less body weight and fat tissue compared to the negative controls.

In summary, the data presented above suggest that loss function of ALCAT1 in mice enhanced the energy expenditure in vivo. Therefore, the mouse ALCAT1 might play a critical regulatory role in inhibiting energy consumption. However, the specific substrate of ALCAT1 in vivo is still missing and the detailed mechanism of ALCAT1 in mice energy metabolism needs further investigation.

\section{Acknowledgements}

The author thanks Professors Kristin Eckert, Cara-Lynne Schengrund and Mala Chinoy (Penn State University) for providing suggestions, guidance and comments on this work. The author also thanks Profs Sara Bronson, Ji-Yue Zhu, Jason Kim and Thomas C. Vary (Penn State University) for their technical support.

\section{References}

Bogdanov M, Mileykovskaya E, \& Dowhan W. (2008) Lipids in the Assembly of Membrane Proteins and Organization of Protein Supercomplexes: Implications for Lipid-Linked Disorders. Subcell Biochem., 49, 197-239.

Bradford B Lowell, \& Spiegelman Bruce M. (2000). Towards a molecular understanding of adaptive thermogenesis. Nature, 404, 652-660.

Barr EL, Zimmet PZ, Welborn TA, Jolley D, Magliano DJ, Dunstan DW, Cameron AJ, Dwyer T, Taylor HR, Tonkin AM, Wong TY, McNeil J, \& Shaw JE. (2007). Risk of cardiovascular and all-cause mortality in individuals with diabetes mellitus, impaired fasting glucose, and impaired glucose tolerance: the Australian Diabetes, Obesity, and Lifestyle Study (AusDiab). Circulation, 116, 151-157.

Bush EN, et al. (2001). Adiposity, Leptin Resistance, Hyperrphagia, Hyperglycemia, Glucose Intolerance and Insulin Resistance in C57BL /6J Mice Fed High Fat Diets. (Metabolic Disease Research, Abbott Laboratories) Endocrine Society Annual Mtg., Poster Session.

Cao J, Liu Y, Lockwood J, Burn P, \& Shi Y. (2004). A novel cardiolipin-remodeling pathway revealed by a gene encoding an endoplasmic reticulum-associated acyl CoA: lysocardiolipin acyltransferase (ALCAT1) in mouse. $J$ Biol Chem, 279, 31727- 31734.

Cao J, Shen W, Chang Z, \& Shi Y. (2009). ALCAT1 is a polyglycerophospholipid acyltransferase potently regulated by adenine nucleotide and thyroid status. Am J Physiol Endocrinol Metab, 296, 647- 653.

Chanseaume E, Tardy AL, Salles J, Giraudet C, Rousset P, Tissandier A, Boirie Y, \& Morio B. (2007) Chronological approach of diet-induced alterations in muscle mitochondrial functions in rats. Obesity (Silver Spring), 15, 50-59.

Dubuc PU, Scott BK, \& Peterson CM. (1993). Sex differences in glycated hemoglobin in diabetic and non-diabetic C57BL/6 mice. Diabetes Res Clin Pract, 21, 95-101.

Eichberg J. (1974). The Reacylation of Deacylated Derivatives of Diphosphatidylglycerol by Microsomes and Mitochondria from Rat Liver. J Biol Chem, 249, 3423-3429.

Gu Z, Valianpour F, Chen S Vaz FM, Hakkaart GA, Wanders RJ, \& Greenberg ML. (2004). Aberrant cardiolipin metabolism in the yeast taz1 mutant: a model for Barth syndrome. Mol Microbiol., 51, 149-158.

Hatch GM. (1998). Cardiolipin: biosynthesis, remodeling and trafficking in the heart and mammalian cells. Int $\mathbf{J}$ Mol Med, 1, 33-41.

Hogan B, Beddington R, Costantini F, \& Lacy E. (1994). Manipulating the Mouse Embryo: A Laboratory Manual. $2^{\text {nd }}$ Edition. New York, USA: Cold Spring Harbor Laboratory. 
Holl MG, Jaser SS, Womack JA, Jefferson VL, \& Grey M. (2010). Metabolic Risk and Health Behaviors in Minority Youth at Risk for Type 2 Diabetes. Diabetes Care. [Epub ahead of print].

Hur YN, Hong GH, Choi SH, Shin KH, \& Chun BG. (2010). High fat diet altered the mechanism of energy homeostasis induced by nicotine and withdrawal in C57BL/6 mice. Mol Cells. [Epub ahead of print].

Jornayvaz FR, Jurczak MJ, Lee HY, Birkenfeld AL, Frederick DW, Zhang D, Zhang XM, Samuel VT, \& Shulman GI. (2010). A high-fat, ketogenic diet causes hepatic insulin resistance in mice despite increasing energy expenditure and preventing weight gain. Am J Physiol Endocrinol Metab. [Epub ahead of print].

Lamson DW, \&Plaza SM. (2002). Mitochondrial factors in the pathogenesis of diabetes: a hypothesis for treatment. Altern Med Rev, 7, 94-111.

Ma L, Vaz FM, Gu Z, Wanders RJ, \& Greenberg ML. (2004). The human TAZ gene complements mitochondrial dysfunction in the yeast taz1Delta mutant. Implications for Barth syndrome. J Biol Chem, 279 (43), 44394-44399.

Malhotra A, Edelman-Novemsky I, Xu Y, Plesken H, Ma J, Schlame M, \& Ren M. (2009). Role of calcium-independent phospholipase A2 in the pathogenesis of Barth syndrome. Proc Natl Acad Sci US A, 106 (7), 2337-2341.

Mancuso DJ, Sims HF, Yang K, Kiebish MA, Su X, Jenkins CM, Guan S, Moon SH, Pietka T, Nassir F, Schappe T, Moore K, Han X, Abumrad NA, \& Gross RW. (2010). Genetic ablation of calcium-independent phospholipase A2 \{gamma\} prevents obesity and insulin resistance during high fat feeding by mitochondrial uncoupling and increased adipocyte fatty acid oxidation. J Biol Chem. [Epub ahead of print].

Neuwald AF. (1997). Barth syndrome may be due to an acyltransferase deficiency. Curr Biol, 7, 465-466.

Parekh PI, Petro AE, Tiller JM, Feinglos MN, \& Surwit RS. (1998). Reversal of diet-induced obesity and diabetes in C57BL/6J mice. Metabolism, 47, 1089-1096.

Rao SS., Disraeli P, \& Mcgregor T. (2002). Impaired Glucose Tolerance and Impaired Fasting Glucose. American Family Physician. 69, 1961-1968.

Riccardi G, Giacco R, \& Rivellese AA. (2004). Dietary fat, insulin sensitivity and the metabolic syndrome. Clin Nutr, 23, 447-456.

Ritz P, \& Berrut G. (2005). Mitochondrial function, energy expenditure, aging and insulin resistance. Diabetes Metab, 31, 5567-5573.

Sambrook J, \& Russell DW. (2001). Molecular Cloning: A Laboratory Manual, 3rd Edition. New York, USA: Cold Spring Harbor Laboratory.

Schlame M, \& Ren M. (2009). The role of cardiolipin in the structural organization of mitochondrial membranes. Biochim Biophys Acta, 1788 (10), 2080-2083.

Schlame M, Rua D, \& Greenberg ML. (2000). The biosynthesis and functional role of cardiolipin. Prog. Lipid Res, 39, 257-288.

Schmidt A, Bickle M, Beck T, \& Hall MN. (1997). The yeast phosphatidylinositol kinase homolog TOR2 activates RHO1 and RHO2 via the exchange factor ROM2. Cell, 88, 531-542.

Schrauwen P. (2007). High-fat diet, muscular lipotoxicity and insulin resistance. Proc Nutr Soc, 66, 33-41.

Sen T, Sen N, Jana S, Khan FH, Chatterjee U, \& Chakrabarti S. (2007). Depolarization and cardiolipin depletion in aged rat brain mitochondria: relationship with oxidative stress and electron transport chain activity. Neurochem Int, 50(5), 719-725.

Shapiro ME. (2001). Effects of Treatment of C57BL/6j Mice Fed High vs. Low Fat Diets with Metformin or Rosiglitazone on Adiposity, Food Intake, Hyperglycemia and Insulin Resistance. (Metabolic Disease Research, Abbott Laboratories). Endocrine Society Annual Meeting, Poster Session.

Testet E, Laroche-Traineau J, Noubhani A, Coulon D, Bunoust O, Camougrand N, Manon S, Lessire R, \& Bessoule JJ. (2005). Ypr140wp, 'the yeast tafazzin', displays a mitochondrial lysophosphatidylcholine (lyso-PC) acyltransferase activity related to triacylglycerol and mitochondrial lipid synthesis. Biochem J, 387, 617-626.

Waqar AB, Koike T, Yu Y, Inoue T, Aoki T, Liu E, \& Fan J. (2010). High-fat diet without excess calories induces metabolic disorders and enhances atherosclerosis in rabbits. Atherosclerosis. [Epub ahead of print]. 
Wiedmer P, Boschmann M, \& Klaus S. (2004). Gender dimorphism of body mass perception and regulation in mice. $J$ Exp Biol, 207, 2859-2866.

Zachman DK, Chicco AJ, McCune SA, Murphy RC, Moore RL, \& Sparagna GC. (2010). The role of calcium-independent phospholipase A2 in cardiolipin remodeling in the spontaneously hypertensive heart failure rat heart. J Lipid Res, 51 (3), 525-534.

Zhao Y, Chen YQ, Li S, Konrad RJ, \& Cao G. (2009). The microsomal cardiolipin remodeling enzyme acyl-CoA lysocardiolipin acyltransferase is an acyltransferase of multiple anionic lysophospholipids. J Lipid Res, 50, 945-956.

Zurlo F, Larson K, Bogardus C, \& Ravussin E. (1990). Skeletal muscle metabolism is a major determinant of resting energy expenditure. $J$ Clin Invest, 86, 1423-1427.

Table 1. High Fat Diet Food consumptions (g/week/mouse)

\begin{tabular}{|c|c|c|c|c|c|c|c|c|c|}
\hline & 1 & 2 & 3 & 4 & 5 & 6 & 7 & 8 & $\begin{array}{c}8 \text { weeks } \\
\text { total(g)/mouse }\end{array}$ \\
\hline M wt & 18.725 & 17.432 & 17.649 & 18.571 & 17.331 & 18.28 & 18.112 & 17.45 & 143.55 \\
\hline M homo & 17.954 & 16.654 & 16.854 & 23.8 & 18.323 & 17.267 & 19.03 & 17.791 & 147.673 \\
\hline F wt & 14.151 & 13.454 & 14.089 & 15.933 & 16.511 & 16.951 & 16.884 & 17.407 & 125.38 \\
\hline F homo & 14.985 & 12.79 & 17.217 & 18.96 & 14.934 & 15.051 & 15.343 & 17.769 & 127.049 \\
\hline
\end{tabular}

Each group size is 5 .

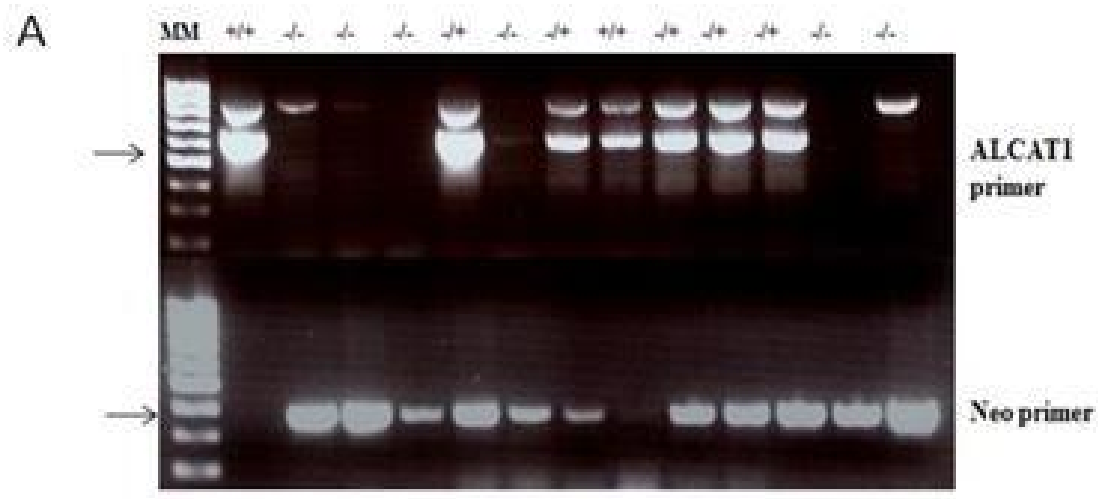

B

WT Heter Homo

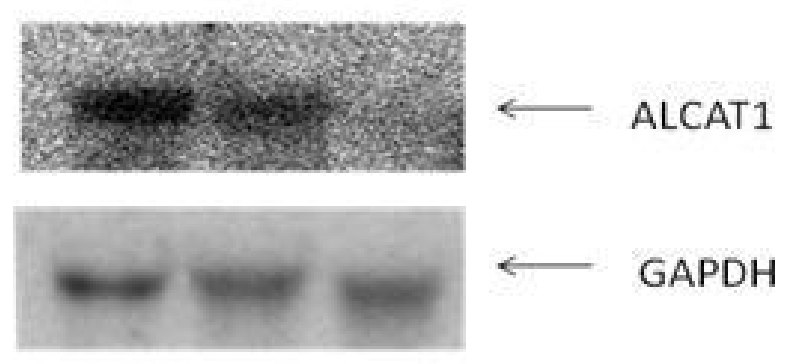

Figure 1. The identification of knockout alcat1 mouse.

A. PCR genotyping with neomycin primers and ALCAT1 primers; B. Northern analysis to check ALCAT1 mRNA expression in mouse liver; probes of Northern blotting: full length of ALCAT1 coding sequence, GAPDH coding sequence fragment as internal control. 
A

body weight with HFD feeding (male)

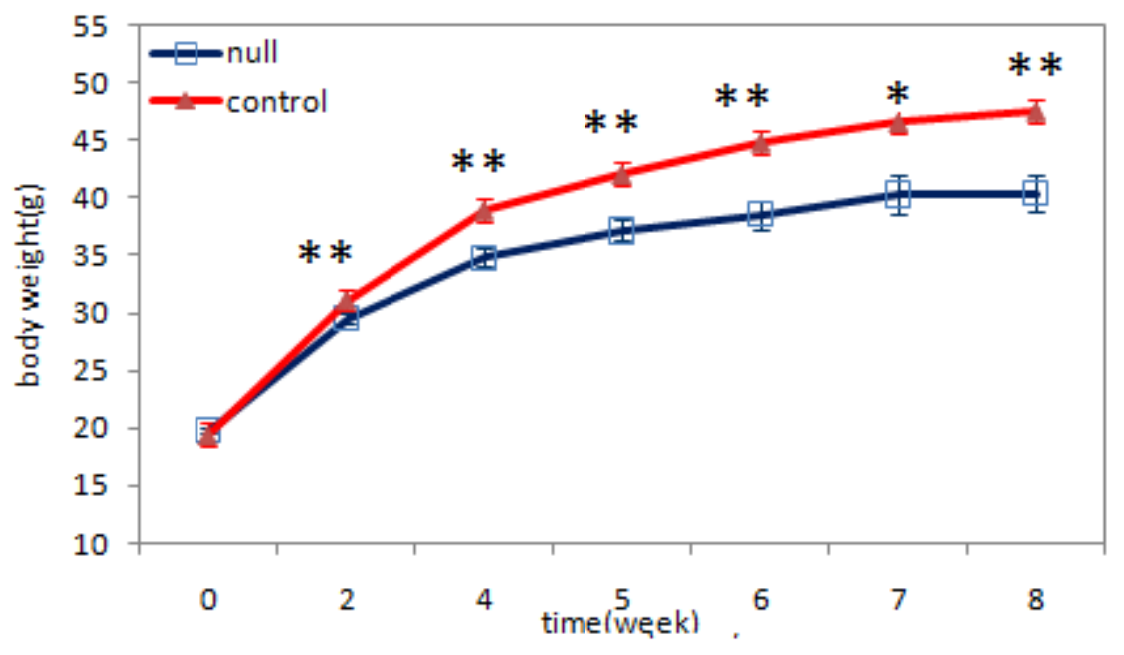

\section{body weight with HFD feeding (female)}

B

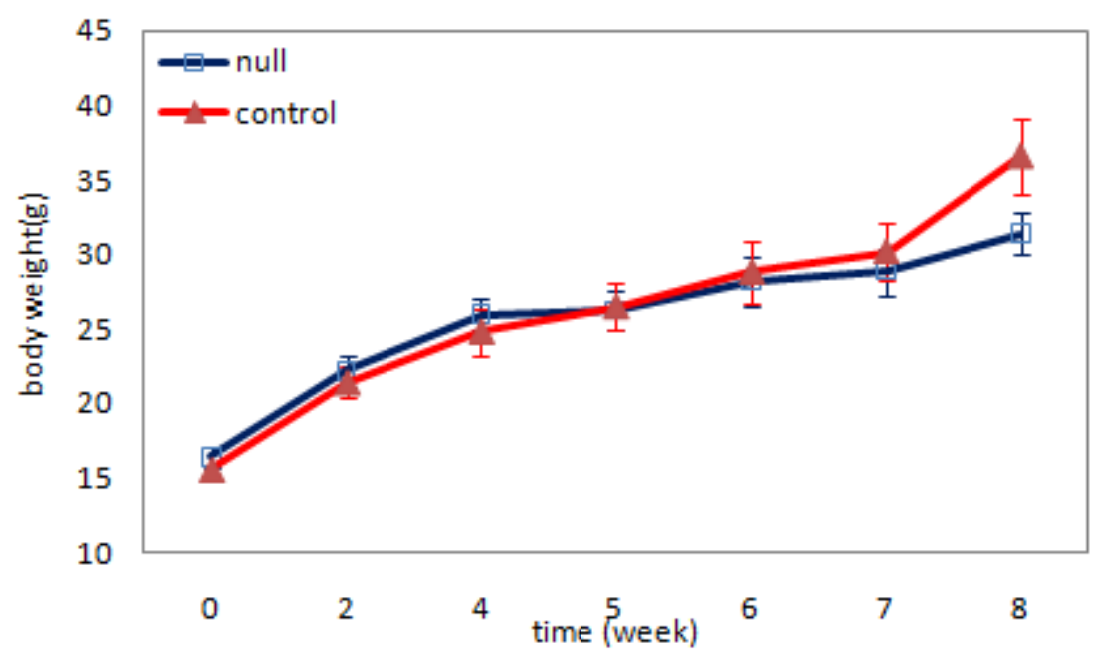

Figure 2. The measurement of mouse body weight with high fat diet feeding.

A. Male mouse; B. Female mouse. --,--- ALCAT1 null mouse; -- - --, control mouse; for each group, the number of tested individual mouse is $4 ;{ }^{*}, \mathrm{P}<0.05 ; * *, \mathrm{P}<0.01$. Error bars are standard errors. 

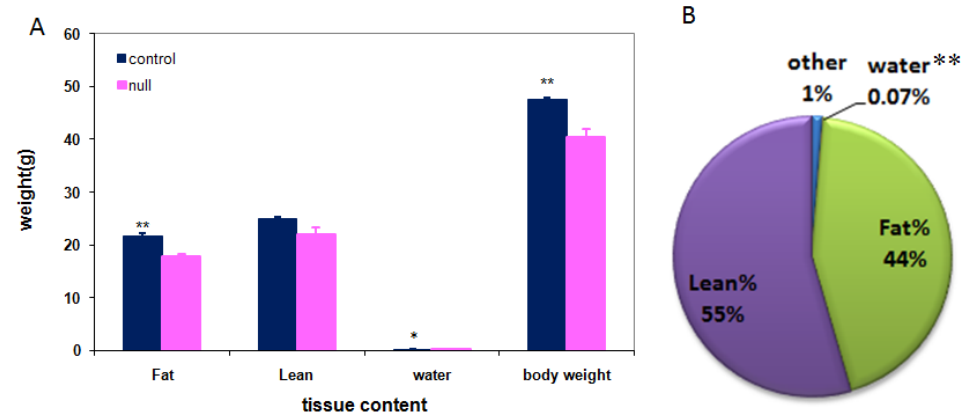

alcat1 KO

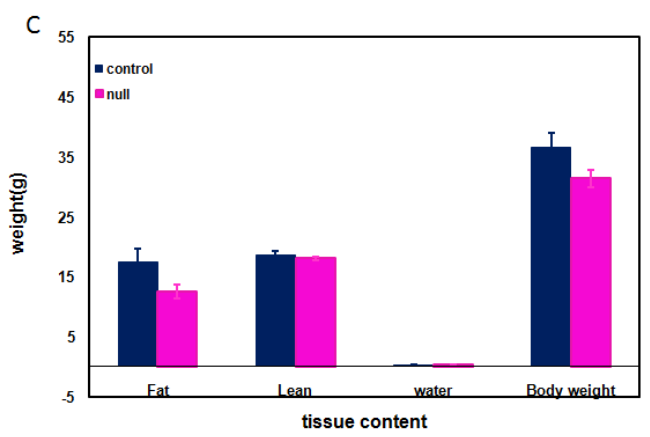

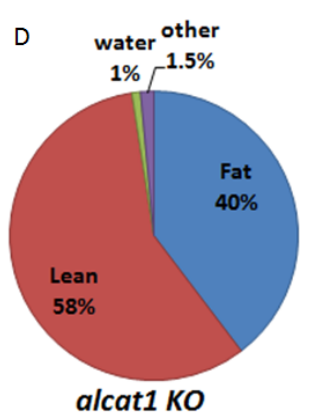

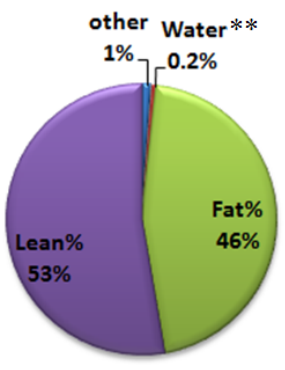

control

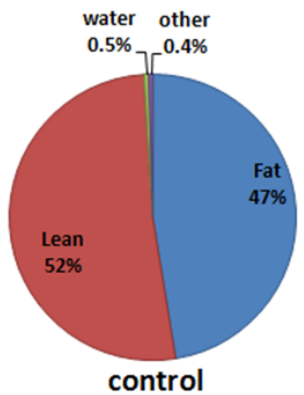

Figure 3. The measurement of mouse tissue content by EchoMRI 3-in-1 after high fat diet challenge.

A. Male mouse tissue content in weight; B. The percentage of male mouse tissue content; $\mathrm{C}$. Female mouse tissue content in weight; D. The percentage of female mouse tissue content. *, $\mathrm{P}<0.05$; **, $\mathrm{P}<0.01$. Error bars are standard error.
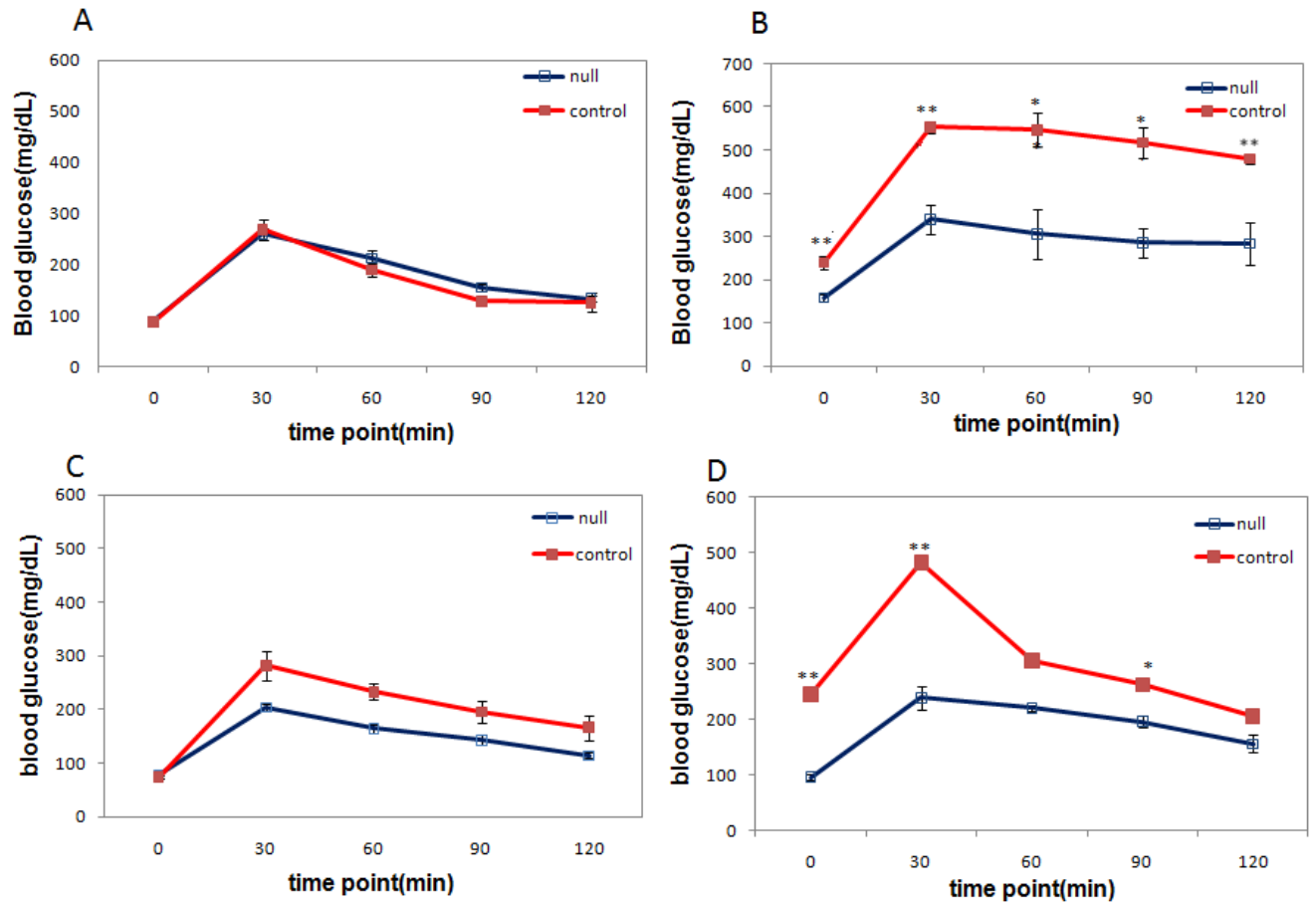

Figure 4. The oral Glucose Tolerance Test (GTT) before and after HFD challenge in mouse.

A. GTT of male mouse before HFD challenge; B.GTT of male mouse after HFD challenge; C.GTT of female mouse before HFD challenge; D. GTT of female mouse after HFD challenge. -- $\square--$, alcat1 null mice; ------, control; for each group, the number of tested mouse is 4; Error bars are standard errors. 
WT + pEMBLyex4

\section{$\Delta \operatorname{taz} 1+\mathrm{pEMBLyex} 4$}

\section{$\Delta$ taz1+FlagALCAT1}

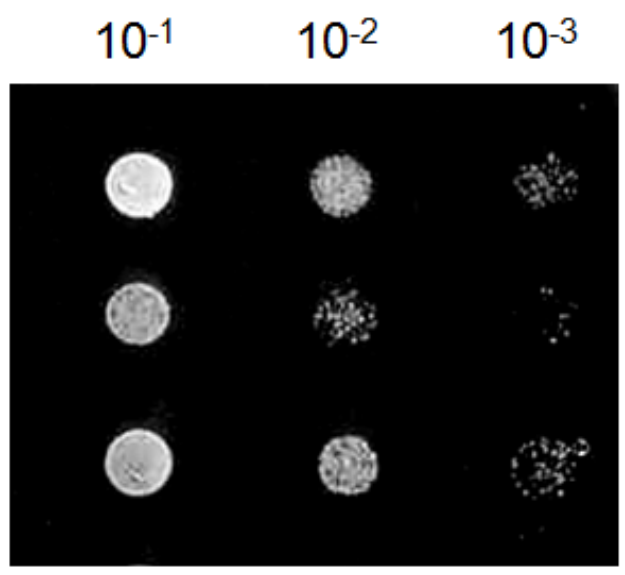

Figure 5. The mouse ALCAT1 gene complemented yeast $\Delta t a z 1$ mutant.

Mouse ALCAT1 transformed in yeast temperature sensitive $\Delta t a z 1$ mutant as described above, complementation experiment was performed at $37^{\circ} \mathrm{C} ; \mathrm{n}=6$.

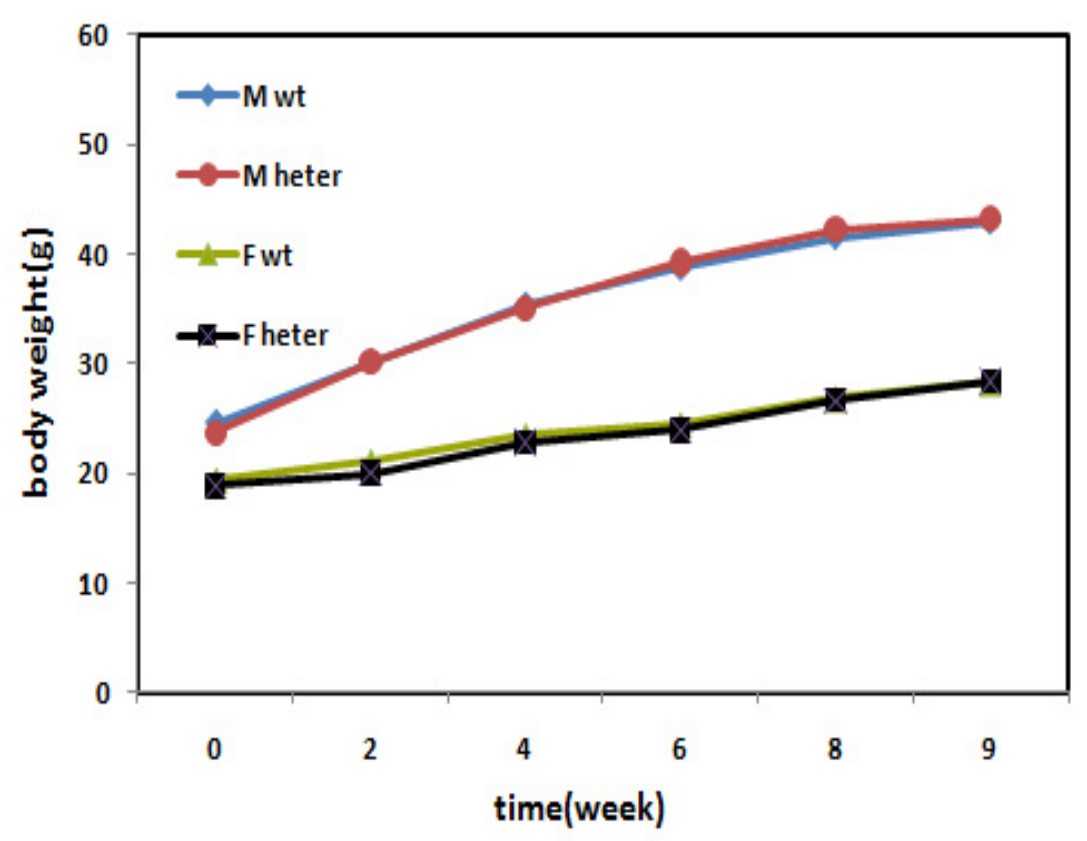

Sup 1 The formula of $60 \%$ high fat diet (D12492).

The Research Diets Inc. provides the $60 \%$ high fat diet (D12492), which can be found on line: http://www.taconic.com/user-assets/Documents/Taconic_D12492.pdf. 
Sup. 2 60\% high fat diet formula (D12492)

\begin{tabular}{|c|c|c|c|}
\hline & & gm\% & Kcal\% \\
\hline Protein & & 26.2 & 20 \\
\hline Carbohydrate & & 26.3 & 20 \\
\hline \multirow{3}{*}{ Fat } & & 34.9 & 60 \\
\hline & Total & & 100 \\
\hline & $\mathrm{kcal} / \mathrm{gm}$ & 5.24 & \\
\hline Ingredient & & gm & Kcal \\
\hline Casein, 80 Mesh & & 200 & 800 \\
\hline L-Cystine & & 3 & 12 \\
\hline Corn Starch & & 0 & 0 \\
\hline Maltodextrin 10 & & 125 & 500 \\
\hline Sucrose & & 68.8 & 275.2 \\
\hline Cellulose, BW200 & & 50 & 0 \\
\hline Soybean Oil & & 25 & 225 \\
\hline Lard* & & 245 & 2205 \\
\hline Mineral Mix S10026 & & 10 & 0 \\
\hline DiCalcium Phosphate & & 13 & 0 \\
\hline Calcium Carbonate & & 5.5 & 0 \\
\hline $\begin{array}{l}\text { Potassium Citrate, } 1 \\
\mathrm{H} 2 \mathrm{O}\end{array}$ & & 16.5 & 0 \\
\hline Vitamin Mix V10001 & & 10 & 40 \\
\hline Choline Bitartrate & & 2 & 0 \\
\hline \multicolumn{4}{|l|}{ FD\&C Yellow Dye \#5 } \\
\hline \multicolumn{4}{|l|}{ FD\&C Red Dye \#40 } \\
\hline FD\&C Blue Dye \#1 & & 0.05 & 0 \\
\hline Total & & 773.85 & 4057 \\
\hline
\end{tabular}

Formulated by E. A. Ulman, Ph.D., Research Diets, Inc., 8/26/98 and 3/11/99.

The body weight of wild type (+/+) and alcatl heterozygote (-/+) littermates in response to high fat diet.

Mice were fed with high fat diet for the indicated period of time and body weight was shown. The mouse numbers in each group is 8 .

*Typical analysis of cholesterol in lard $=0.95 \mathrm{mg} / \mathrm{gram}$. 